\title{
TREATMENT OF SECONDARY LOWER LIMB LYMPHEDEMA AFTER GYNECOLOGIC CANCER WITH COMPLEX DECONGESTIVE THERAPY
}

\author{
F. Liu, N. Liu, L. Wang, J. Chen, L. Han, Z. Yu, D. Sun
}

Department of Plastic \&Reconstructive Surgery, Shanghai Ninth People's Hospital, Shanghai Jiao Tong University School of Medicine, Shanghai, China

\section{ABSTRACT}

Secondary lower extremity lymphedema is a common complication of treatment for gynecological cancers. Conservative therapy plays an important role in the treatment of patients with secondary lower extremity lymphedema; in particular, complex decongestive therapy(CDT) has been recognized as an effective nonoperative technique for these patients. But CDT therapy for secondary lower extremity lymphedema remains a problem in China because this technique and its effectiveness have not achieved widespread use and popularity. Our goal was to assess effects of CDT in patients with secondary lower limb lymphedema after treatment for gynecological cancers. The retrospective study consisted of 60 patients who were treated with 20 sessions of CDT. Assessments included objective changes in limb circumference, degree of $L E$, imaging features, and incidence of erysipelas before and after CDT treatment. We found that CDT can effectively improve lymph stasis and promote backflow, and decrease circumference, interstitial fluid content, and incidence of erysipelas of lymphedematous lower limb. Our results demonstrate that CDT is an effective treatment method for patients with secondary lower limb lymphedema following treatment for gynecologic cancers. This technique should be more widely utilized and popularized in China to improve the quality of life of millions of patients with secondary lower limb lymphedema.

Keywords: lymphedema, gynecologic cancer, complex decongestive therapy, manual lymphatic drainage, bandage compression therapy, functional exercise

Lymphedema (LE) is a chronic disease caused by lymphatic system dysplasia or damage. It is characterized by an imbalance in lymphatic flow that leads to accumulation of protein-rich fluid in the interstitium of tissues (1). LE is etiologically classified into primary and secondary LE (SLE) (2). SLE has known causes such as radiotherapy, trauma, filariasis, and radical cancer operations (3). Secondary lower extremity LE is a common complication of treatment for gynecological cancers including cervical, ovarian, endometrial, and others. Both radical surgery and radiotherapy, which may damage the lymph nodes and lymphatic vessels involved in lymph drainage routes, are implicated in the development of lower extremity LE (4).

SLE presents as chronic swelling of the lower limbs and is frequently accompanied by limb pain, skin thickening, hyperpigmentation, and altered sensation (5). If untreated, patient are at increased risk of infection, skin fibrosis, and functional limitations of the affected limb. Additionally, patients with LE 
may experience significant psychosocial distress and poor quality of life (6). Thus, it is especially important to reduce swelling and relieve symptoms in patients with SLE (7). Conservative therapy plays an important role in the treatment of patients with LE; in particular, complex decongestive therapy (CDT) has been recognized as an effective nonoperative technique for patients with LE and is recommended by the International Society of Lymphology $(8,9)$.

CDT is a comprehensive treatment that combines manual lymphatic drainage (MLD), bandage compression therapy, functional exercise, and meticulous skin care (10). This therapy is carried out in two phases. The aim of the first treatment phase is to mobilize the accumulated lymph, soften the fibrotic tissue, and improve the health of the skin using daily MLD and multilayered bandage wrapping. In the second maintenance phase, compression by low-stretch elastic bandages or fabrics and repeated light massage are performed to conserve and optimize the results obtained in the first phase. The patients are also instructed in how to care for the skin and exercise in daily life $(10,11)$. MLD is a major component of CDT and used to redirect lymph across the plexus of initial lymphatics toward healthy lymph nodes by manual massage (12). After MLD, a set of low stretch bandages is used to maintain the volume reduction and can provide appropriate pressure during rest and contraction of limb muscles to increase lymph flow (13). Skin care is also important to prevent infection and ulceration. Appropriate exercise with bandage protection is beneficial to contract the limb muscles and thus facilitate lymph flow.

Treatment for LE remains a problem in China because the CDT technique and its effectiveness have not achieved widespread use and popularity. Few institutions or hospitals perform CDT for patients with LE, leading many patients to irrationally choose surgical therapy, acupuncture, and cupping therapy, resulting in irreversible limb damage. Nevertheless, we advocate and perform CDT at our Lymphedema Therapy Centre. We herein report the results of a retrospective study on the treatment of lower limb SLE using CDT to promote lymph return and reduce limb swelling in patients after treatment for gynecological cancers.

\section{MATERIALS AND METHODS}

\section{Ethics Approval and Consent to Participate}

This retrospective study was undertaken at the Shanghai Ninth People's Hospital. The retrospective analysis was approved by the Human Ethics Review Committee as well as the Helsinki Declaration. Written informed consent was obtained from all patients for the use of their clinical data.

\section{Study Design}

After informed consent, all patients were treated with 20 sessions of CDT by an experienced and educated LE therapist using MLD, bandage compression therapy, and skin care, with therapist instructions for patients to continue physical exercise at home. Assessments included objective changes in limb circumference, the degree of LE, and imaging features. Assessments were performed before CDT and at the end of the treatment program. Additionally, the incidence of erysipelas was compared before CDT and 1 year after CDT by telephone follow-up.

\section{Patients}

Sixty female patients (average age, 55.1 years; range, 38-76 years) with secondary unilateral lower limb LE were enrolled in this study from July 2017 to May 2019. All patients had undergone radical surgery and completed radiotherapy and/or chemotherapy sessions for endometrial cancer, ovarian cancer, or cervical cancer. The average duration of SLE was 4.06 years, and the SLE ranged from stage I to IV. The left limb was edematous in 41 patients, and the right was edematous in 19. No patients had cancer recurrence, deep venous thrombosis of the limbs, kidney failure, or cardiac edema according to examination of blood biochemistry testing and medical imag- 


\begin{tabular}{|c|c|}
\hline $\begin{array}{r}\text { TA } \\
\text { Characteristics } \\
\text { Part }\end{array}$ & $\begin{array}{l}\text { E } 1 \\
\text { anrolled Study } \\
\text { ants }\end{array}$ \\
\hline Patient characteristics & $\begin{array}{l}\text { Secondary lower limb } \\
\text { lymphedema }(n=60)\end{array}$ \\
\hline Age (average, range) & $55.1(38-76)$ \\
\hline Gender & \\
\hline Female & 60 \\
\hline Male & 0 \\
\hline LE stage & \\
\hline I & 5 \\
\hline II & 24 \\
\hline III & 26 \\
\hline IV & 5 \\
\hline Duration of LE, yr & $4.06(0.1-16)$ \\
\hline Type of gynecological $n$ & gnancy \\
\hline Cervical cancer & 38 \\
\hline Ovarian cancer & 9 \\
\hline Endometrial cancer & 13 \\
\hline Affected unilateral low & $\mathbf{m b}$ \\
\hline Left leg & 41 \\
\hline Right leg & 19 \\
\hline
\end{tabular}

ing performed by oncologists and other physicians. The detailed characteristics of all patients are presented in Table 1.

\section{Treatment}

MLD was performed using a modified method described by Földi (14). Before MLD, the patient cleaned her lower limbs and then assumed a supine position with her lower limbs extended horizontally. The certified LE therapist gently massaged the lymph nodes, lymphatic vessels, and skin involved in the lymph drainage areas using different manual techniques, including the stationary circle, rotary, pump, and scoop techniques. The manual drainage was directed toward the functional lymph nodes to mobilize the lymphatic fluid from congested regions to normal tissue $(15,16)$. First, in the preparation period, the therapist effleuraged the bilateral supraclavicular, axillary, and inguinal lymph nodes and the abdominal lymphatic trunk to stimulate them to drain stagnated lymph. Based on the routes of lymph backflow in the lower limb, the therapist then effleuraged the skin area from the proximal aspect (thigh) to the distal segment (foot), to activate intradermal initial lymphatic vessels and subcutaneous collectors, promoting reabsorption of stagnated lymphatic fluid in the interstitial space. Third, massage was applied to the edematous limb from thigh to groin and from foot to leg. Finally, the whole limb was massaged from the distal to proximal extremity, promoting lymphatic fluid backflow through functional lymphatic vessels. After CDT, sweat was toweled off the limb to keep the skin clean and dry, and suitable emollients were applied to the skin. The therapist then applied various types of bandages for compression therapy. A long tubular cotton bandage was directly applied to cover the whole affected lower limb. An elastic gauze bandage was then loosely applied in an initial anchoring turn around the forefoot at the metatarsal joint, first wrapping the big toe and then bandaging the other four toes. The therapist spirally bandaged a polyurethane pad from foot to thigh to reduce friction and buffer pressure, ensuring greatest thickness of the pad on the ankle and knee. Finally, the therapist used a low-stretch bandage to compress the whole limb by a "figure-of-eight" overlapping technique, instructed the patient in exercising (walking, flexing the leg) for 5 minutes, and then checked the pressure and tightness of the compression bandages to ensure that they were not causing ischemia, tingling, or a pins-and-needles sensation (Fig. 1). The whole CDT session included $30 \mathrm{~min}$ of MLD, 20 min of multilayer bandage compression therapy, skin care, and $\mathbf{1 0} \mathrm{min}$ of exercising. The entire CDT session was performed once per day, and the treatment course comprised 20 sessions. Every patient underwent one treatment course.

\section{Measurement and Analysis of Limb Circumference}

Each edematous limb was assessed using a standard 1-mm fiber tape to measure the circumference. For lower limb circumference measurements, the patients lay in the supine position with their legs relaxed and knees straight. The circumference was measured at five positions on the lower limb: $5 \mathrm{~cm}$ above the second plantar toe joint, $5 \mathrm{~cm}$ above the 


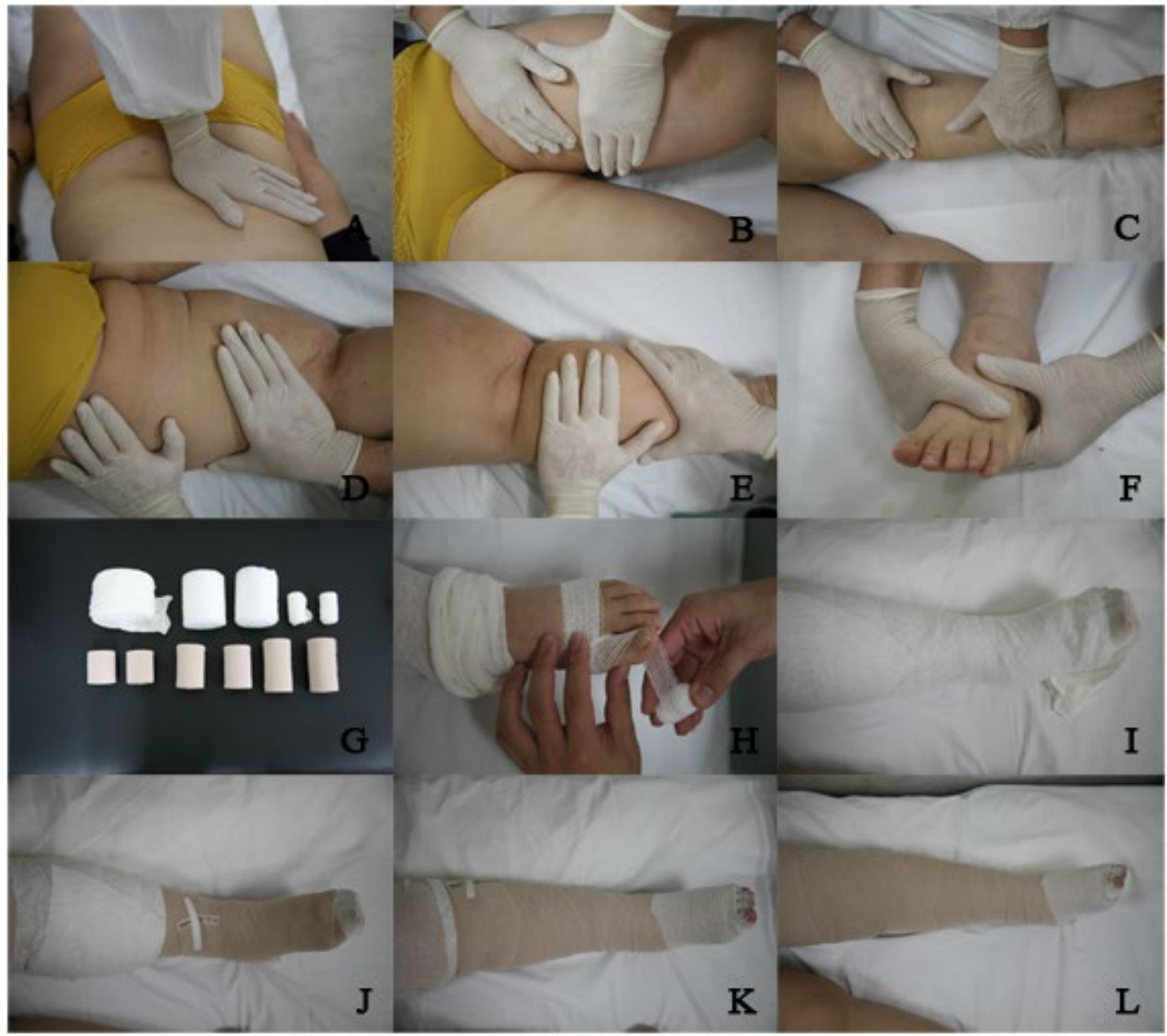

Fig. 1. Technique of CDT applied to study subjects. (A-F) MLD with application from the trunk to the toes. (G) Bandaging materials include cotton bandages, polyurethane pads, and short-stretch elastic bandages. $(H-L)$ Application of bandaging from the toes to the upper leg.

with their legs relaxed and knees straight. The circumference was measured at five positions on the lower limb: $5 \mathrm{~cm}$ above the second plantar toe joint, $5 \mathrm{~cm}$ above the lateral malleolus, $10 \mathrm{~cm}$ below the lower border of the patella, 10 $\mathrm{cm}$ above the superior border of the patella, and $20 \mathrm{~cm}$ above the superior border of the patella. We compared the circumference ratio before and after CDT to calculate the mean circumference ratio of the five positions and analyzed the change in the edematous limb after treatment. All measurement data were recorded in centimeters $(\mathrm{cm})$. The circumfer- ence ratio was defined as follows: (mean circumference value of lymphedematous limb / mean circumference value of contralateral limb) / mean circumference value of contralateral limb.

\section{Measurement and Analysis of Interstitial Fluid Content of the Limb}

We measured and analyzed the interstitial fluid content of the lower limbs using a multiple-frequency bioelectrical impedance analysis device (Inbody 3.0; Biospace Co. Ltd., 
Seoul, Korea). This machine uses the impedance of tissue fluid to tiny electrical currents $(1 \mathrm{KHz}-1 \mathrm{MHz})$ to accurately indicate the interstitial fluid change in the edematous limb. We compared the interstitial fluid ratio before and after CDT to assess the effect of CDT. All measurement data were recorded in kilogram (kg). The interstitial fluid ratio was defined as follows: (mean interstitial fluid value of lymphedematous limb / mean interstitial fluid value of contralateral limb) / mean interstitial fluid value of contralateral limb.

\section{Indocyanine Green Lymphography}

Indocyanine green (ICG) lymphography was performed on 10 patients with SLE. The patients were placed supine on a bed. After intradermal injection of $0.25 \mathrm{mg} / \mathrm{ml}$ of ICG into the toe web spaces (three points for each dorsum of the foot, $0.05 \mathrm{ml}$ at each point), a photodynamic eye fluorescence-locating instrument (PDE; Hamamatsu Photonics, Hamamatsu, Japan) was used for dynamic observation of lymph flow 30 min after injection. Sequential fluorescence real-time imaging of the lymphatics was thus acquired $(17,18)$.

\section{Assessment of Incidence of Erysipelas}

We recorded and compared the incidence of erysipelas before CDT and 1 year after CDT by telephone follow-up to assess the effect of CDT.

\section{Statistical Analysis}

Data are presented as mean (range) or number (percent). Statistical analysis was performed using T-test with SPSS 17.0 software (IBM, Armonk, NY, US). All tests were two-sided, with a significance level of 0.05 or 0.01 .

\section{RESULTS}

\section{Decreased Limb Circumference after CDT}

The circumference of the lymphedematous limbs was significantly smaller after CDT
(Fig. 2). Pre-treatment, circumference, and circumference ratio of the lymphedematous limbs were $39.8 \pm 5.14 \mathrm{~cm}$ and $0.199 \pm 0.1529$, respectively. After CDT, circumference and circumference ratio of the lymphedematous limbs had decreased to $35.307 \pm 4.1468 \mathrm{~cm}$ and $0.058 \pm 0.0474$, respectively, which were both significantly smaller $(\mathrm{p}<0.01)$ (Fig. 3).

\section{Decreased Interstitial Fluid Content after $C D T$}

The interstitial fluid content was obviously reduced in lymphedematous limbs after CDT. Pre-treatment, interstitial fluid content and ratio were $7.6 \pm 1.97 \mathrm{~g}$ and $0.481 \pm 0.0428$, respectively. After CDT, interstitial fluid content and ratio had decreased to $5.6 \pm 0.89 \mathrm{~g}$ and $0.131 \pm 0.0937$, respectively, which were both significantly lower $(\mathrm{p}<0.01)$ (Fig. 4).

\section{Improved Lymph Stasis and Dermal Backflow after $C D T$}

ICG lymphography images of lymph flow and lymphatic vessels showed significant differences in the lymphatic vasculature between the control limbs and affected limbs. The lymphatic structure in the normal limbs generally consisted of well-defined channels that actively propelled lymph flow to the inner part of the leg and thigh and drained it into the inguinal nodes (data not shown). In contrast, the lymphatic structure in the lymphedematous limbs typically displayed substantial lymph fluid stasis in dilated and tortuous lymphatic vasculature. Some seriously affected lower limbs showed dermal backflow and lymphatic fluid leakage. After CDT, the degree of lymph fluid stasis and dermal backflow decreased, and well-defined, normal lymphatic vessels were observed in some improved regions (Fig. 5).

\section{Decreased Incidence of Erysipelas after CD}

The average annual incidence of erysipelas in lymphedematous limbs was lower after than before CDT. Pre-treatment, average annual incidence of erysipelas was $0.522 \pm$ 


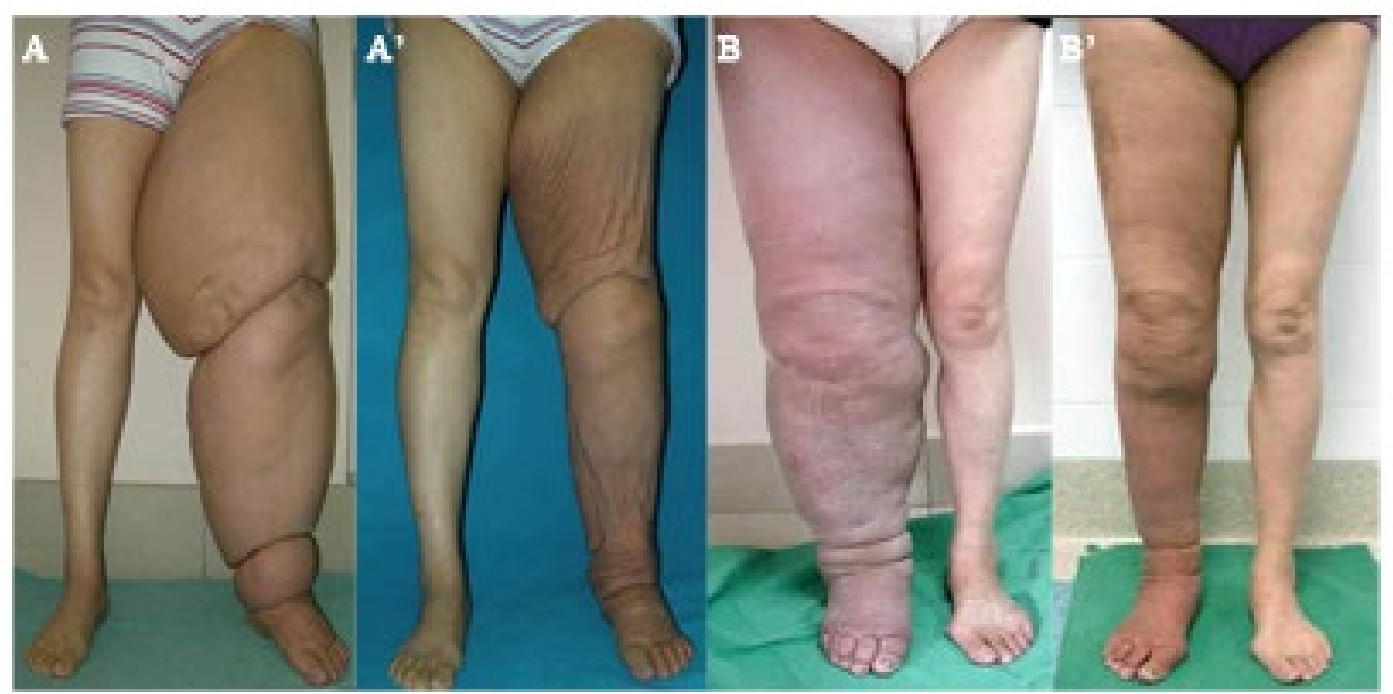

Fig. 2. Two subjects demonstrating effectiveness of CDT treatment in reducing lymphedema pre $(A, B)$ and post $\left(A^{\prime}, B^{\prime}\right)$.
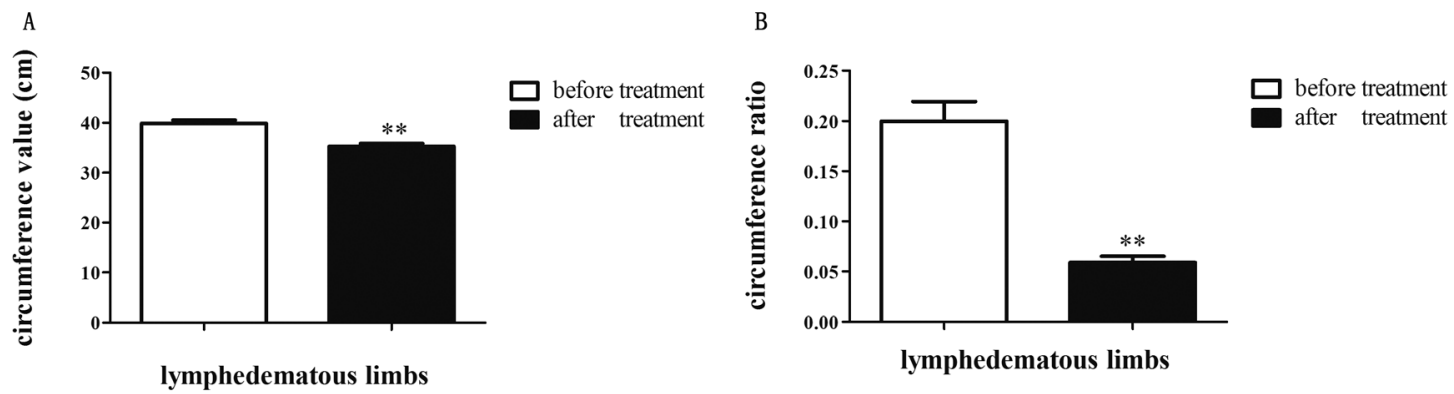

Fig. 3. Circumference ( $A$ ) and circumference ratio (B) were both significantly reduced ( $p<0.01)$ after $C D T$ treatment.

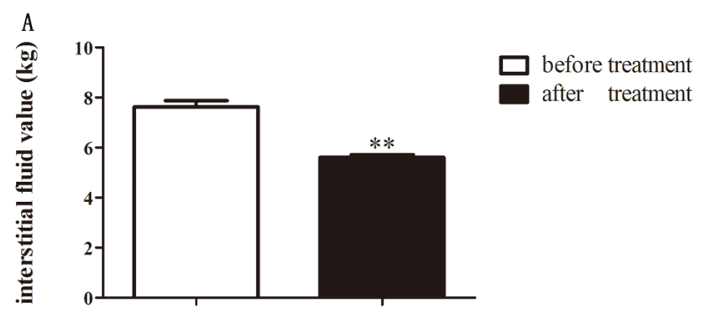

lymphedematous limbs

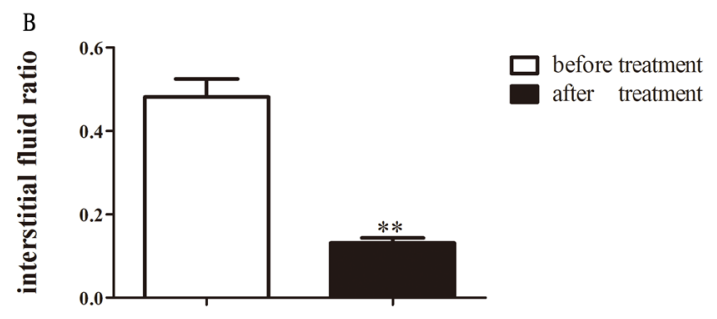

lymphedematous limbs

Fig. 4. Interstitial fluid value $(A)$ and interstitial fluid ratio $(B)$ were both significantly reduced $(p<0.01)$ after $C D T$ treatment. 


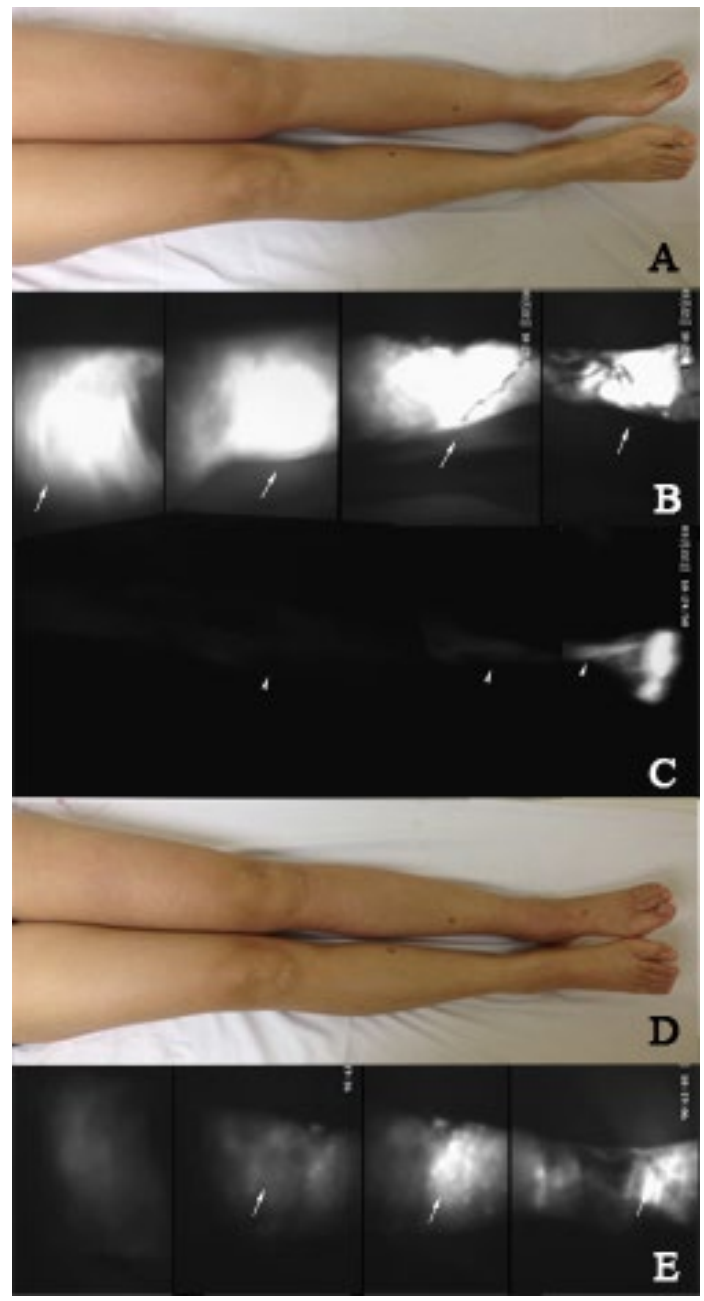

Fig. 5. Patient images of one subject pre (A) and post (D) with corresponding ICG Images. (B) PreCDT ICG image displays lack of identifiable channels and substantial dermal diffusion. (C) ICG image displays the identifiable lymphatic channels in normal lower limb. (E) Post-treatment imaging depicts identifiable vessel imaging as well as a substantial reduction in dermal diffusion.

0.1245. After CDT, average annual incidence had decreased to $0.216 \pm 0.0633$, which was significantly lower $(\mathrm{p}<0.01)$ (Fig. 6).

\section{DISCUSSION}

In this study, we evaluated the efficacy of CDT in treating the lower limbs of patients with SLE due to gynecological cancer. Our results demonstrate that CDT can improve the function of lymphatic vessels, reduce backflow of stagnated lymph, and significantly reduce both interstitial fluid content and circumference of lymphedematous lower limbs. CDT can also enhance the anti-infection capabilities of the skin, decreasing the incidence of erysipelas. Therefore, CDT may play an important role in the treatment of lower limb SLE, and its use and popularization in China would be beneficial.

Lower extremity SLE is a refractory medical condition that occurs in $1 \%$ to $49 \%$ of cancer survivors, and it is a chronic condition requiring long-term, even lifelong treatment (19-21). Therapy is currently divided into operative and conservative (nonoperative) treatment (8). Operative treatments include autologous lymph vessel transplantation, lymphovenous shunting, "debulking" operations, and others; however, these techniques have not become widely popular because of their strict indications, sophisticated microsurgical manipulations, and uncertain long-term effects. Conversely, conservative treatment has played the key role in patients with SLE and is recommended by several groups of experts because of its noninvasive manipulation and fewer complications. Some special conservative therapies are used in China, such as Chinese herbs, heat and bandage treatment, and Qigong exercises (22-24). CDT is a promising technique recommended by the International Society of Lymphedema and has already been widely employed in Europe and North America. This technique was first introduced to China by Ningfei Liu in 2007 and has since been gradually promoted (25).

MLD was first introduced by Emil Vodder to treat edematous lymph nodes in 1930 (26), and later adding the bandage compression therapy, functional exercise, and skin care named CDT by Földi (27). MLD is a light, hands-on therapy designed to reduce lymph swelling by enhancing lymphatic drainage, and it is administered as the most important part of CDT. The manipulative principle of MDL is based on the anatomy and physiology of the lymphatic system. Unlike physiology of 


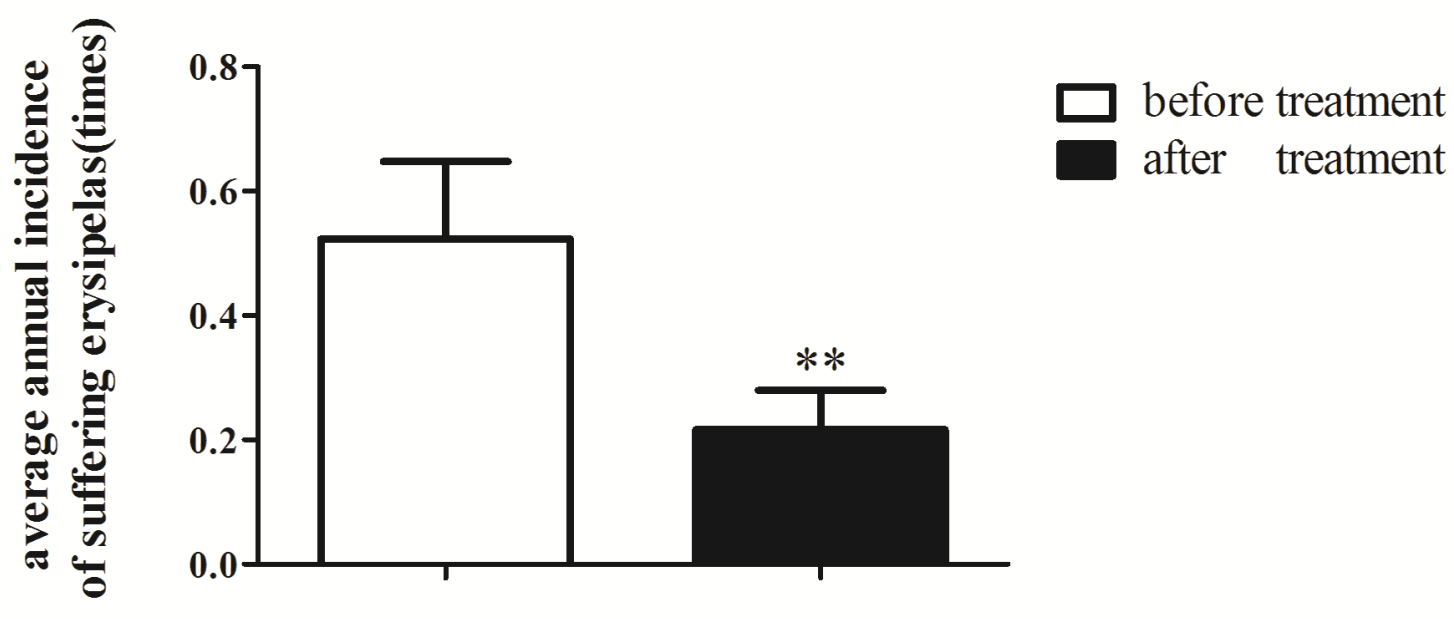

\section{lymphedematous limbs}

Fig. 6. Significant reduction $(p<0.01)$ in average annual incidence of erysipelas pre- and post-treatment for all 60 subjects. The incidence of erysipelas was recorded and compared in 60 patients before CDT and 1 year after CDT by telephone follow-up to assess the effect of CDT.

the lymphatic system. Unlike the blood, which is propelled through the body by the dynamic pumping of the heart, lymphatic fluid is moved by the rhythmic contractions of the muscular walls of lymphatic vessels. The sequential, segment-to-segment contractions of the lymphatic vessels are under the control of the autonomic nervous system but can be regulated by external stimuli on the lymphatic vessels, such as that from compression of surrounding muscles or local arterial pulsation (28). In the present study, as shown by ICG lymphography images of lower limb SLE, the lymphatic vessels of the affected leg and popliteal lymph nodes were not completely damaged, but the pelvic lymphatic system was damaged, resulting in drainage of interstitial fluid and obstruction. MLD can stimulate peristaltic contractions in these affected lymphatic vessels. The initial lymphatic vessels respond to the rhythmic massage movement, opening to allow more fluid to enter the lymphatic system. It is also believed that MLD can influence the contractile function of the collector lymphatic vessels, enhancing lymph flow and creating a suction effect that pulls lymph from distal areas toward the lymph nodes (12). By this treatment, swelling is reduced by pumping of lymphatic vessels, reabsorption of lymph, a reduction in hydrostatic resistance to lymph flow, and a rerouting of lymph away from areas of stasis and into viable lymphatic vessels (29). This results in a significant reduction in the circumference and interstitial fluid content of edematous limbs after an MLD treatment course.

After MLD, compression therapy using different types of bandages is essential. A multi-layered compression bandage is applied and overlapped on the lower limb from foot to thigh. The bandage produces the appropriate pressure on the skin to help reduce interstitial fluid by decreasing its formation and preventing lymph refill into the interstitial spaces that have been drained by MLD to maintain volume reduction (30). Compression also assists limb muscle contraction to create a "muscle pump" effect that increases the lymph and blood flow during rest and exercise. These bandages should be short stretch, which exerts a relatively low pressure on the limb at rest and cannot affect movement of the limb. 
Additionally, when the limb is in motion and the muscles are contracting, short-stretch bandages maintain their integrity as the muscles contract against the bandages and provide a semi-rigid support structure for the muscles to contract against working pressure; this reduces capillary filtration, resulting in further edema reduction (31). We use low-stretch compression bandages for treatment of SLE and instruct the patients in how to use the bandage to perform treatment at home. However, some patients cannot carry out this treatment by themselves; in such cases, we advise them to use a compression stocking or customfitted garment that is worn during the day to maintain adequate compression on their edematous limbs $(10,32)$.

Skin care should also be performed during CDT. The patients should keep their edematous skins clean and dry, and they should not walk barefoot at any time to avoid accidental injury or insect bites; this will reduce the risk of infection and ulceration of the limbs. Suitable emollients can be applied to the skin to prevent hyperkeratosis and eczema. In addition, therapists and patients should sanitize their hands or wear medical latex gloves when contacting the edematous skin (33). Patients with LE should be taught and encouraged to perform low- to moderateintensity physical exercise. Proper exercise with bandage protection is beneficial for limb muscle contraction; this prevents additional swelling and facilitates lymphatic flow. However, overstretching and high-intensity exercise should be avoided $(34,35)$.

In the present study, we treated lower limb SLE using CDT instead of surgery. We carefully executed the treatment process for all patients and taught them to perform the technique by themselves. All patients were satisfied with the effects of CDT; in particular, they noted the reduction of the circumference and interstitial fluid content of the affected limb. This result is consistent with previous research. Williams et al. conducted a randomized controlled crossover study and found that CDT significantly reduced the limb volume and circumference (36). Weiss reported that patient satisfaction after CDT appeared to be associated with the limb volume reduction and recovery of the normal limb appearance (37). Furthermore, improved lymphatic vessel function was observed using ICG lymphography. The PDE system showed that the high-intensity area of the fluorescent signal around the lymphedematous limb was reduced and that the low-intensity signal was increased. These changes indicate that the application of CDT to the edematous limb encouraged lymph fluid circulation. Thus, the efficacy of CDT was visualized, and the fluorescent images were well supported by the changes in circumference and interstitial fluid. Tan et al. suggested that MLD is a viable treatment for LE using ICG fluorescence imaging to demonstrate the improvement of lymphatic vessel function (17). Takeno et al demonstrated the efficacy of CDT on edematous rat limbs by real-time ICG lymphography (38). Additionally, it is possible that CDT can eliminate many infiltrative microbes and specific antigens by improving lymph backflow, stimulating lymphatic reabsorption, and decreasing tissue edema; this may decrease the incidence of cellulitis and erysipelas of the lower limb (39).

Based on the treatment effects and understanding of the CDT technique, we believe that this technique has some advantages over other types of treatments. CDT has very remarkable effects on lower limb SLE by restoring the function and appearance of the limb as well as improving quality of life. Furthermore, in contrast to surgical manipulation of the lymphatic nodes and vessels, CDT is noninvasive and does not damage the skin structures, thus reducing patient anxiety and complications such as scarring, ulceration, and lymphorrhagia. This technique can also be adapted to both adults and children and is easy to learn and perform for long-term home care. Finally, CDT can be used to alleviate symptoms in special lymphedematous sites such as the genitalia and toes.

Although this study population was limited to patients with lower limb lymphedema following gynecological cancer, there is no reason why it cannot be applied to patients with other causes of lower limb lymphedema. Further studies to confirm these results in 
other setting as well as with other causes of lymphedema can strengthen these results.

In the present study, we have illustrated that CDT is an effective treatment method for patients with SLE following gynecological cancer treatment. Therefore, the CDT technique should be more widely utilized and popularized in China to improve the quality of life of millions of patients with lower limb SLE.

\section{CONFLICT OF INTEREST AND DISCLOSURE}

The authors declare no competing financial interests exist.

\section{ACKNOWLEDGMENT}

This study was supported by Chinese National Science Foundation (grant number 81272146).

\section{REFERENCES}

1. Chiu, TW: Management of secondary lymphedema. Hong Kong Med. J. 20 (2014), 1-10.

2. Connell, F, G Brice, S Jeffery, et al: A new classification system for primary lymphatic dysplasias based on phenotype. Clin Genet. 77 (2010), 438-452.

3. Warren, AG, H Brorson, LJ Borud, SA Slavin: Lymphedema: A comprehensive review. Ann. Plast. Surg. 59 (2007), 464-472.

4. Franks, PJ, CJ Moffatt, DC Doherty, et al: Assessment of health-related quality of life in patients with lymphedema of the lower limb. Wound Repair Regen. 14 (2006),110-118.

5. Leung, EYL, SA Tirlapur, C Meads: The management of secondary lower limb lymphoedema in cancer patients: A systematic review. J. Palliat. Med. 29 (2015), 112-119.

6. Dawes, DJ, S Meterissian, M Goldberg: Impact of lymphoedema on arm function and health-related quality of life in women following breast cancer surgery. J. Rehabil. Med. 40 (2008), 651-658.

7. Zhang, L, A Fan, J Yan, et al: Combining manual lymph drainage with physical exercise after modified radical mastectomy effectively prevents upper limb lymphedema. Lymph. Res. Biol. 1 (2016), 1-5.
8. International Society of Lymphology. The diagnosis and treatment of peripheral lymphedema: 2020 Consensus Document of the International Society of Lymphology. Lymphology 53 (2020), 3-19.

9. Boris, $M, S$ Wendorf, B Lasinski: Lymphedema reduction by noninvasive complex lymphedema therapy. Oncology 8 (1994), 95-106.

10. Ezzo J, E Manheimer, ML McNeely, et al: Manual lymphatic drainage for lymphedema following breast cancer treatment (Review). The Cochrane Collaboration. JohnWiley \& Sons, Ltd. 2015.

11. Badger, C, C Preston, K Seers, $P$ Mortimer: Physical therapies for reducing and controlling lymphoedema of the limbs. Cochrane Database of Systematic Reviews 4 (2004), 1-36.

12. Williams, A: Breast and trunk oedema after treatment for breast cancer. J. Lymphoedema 1 (2006), 32-39.

13. Tsai, HJ, HC Hung, JL Yang, et al: Could kinesio tape replace the bandage in decongestive lymphatic therapy for breastcancer related lymphedema? A pilot study. Supportive Care in Cancer. 17 (2009), 13531360.

14. Földi, M, E Földi. Földi's Textbook of Lymphology for Physician and Lymphedema Therapists. New York: Urban and Fischer, 2012

15. Liu, NF: Liu NF's Textbook of Lymphedema Diagnosis and Treatment. Chinese Science Press, 2014.

16. Gurdal, SOA Kostanoglu, I Cavdar, A Ozbas, et al: Comparison of intermittent pneumatic compression with manual lymphatic drainage for treatment of breast cancer-related lymphedema. Lymphat. Res. Biol. 3 (2012),129-135.

17. Tan, I-C, EA Maus, JC Rasmussen, et al: Assessment of lymphatic contractile function following manual lymphatic drainage using near-infrared fluorescence imaging. Arch. Phys. Med. Rehabil. 92 (2011), 756-764.

18. Yamamoto, T, N Matsuda, K Doi, et al: The earliest finding of indocyanine green lymphography in asymptomatic limbs of lower extremity lymphedema patients secondary to cancer treatment: The modified dermal backflow stage and concept of subclinical lymphedema. Plast. Reconstr. Surg. 128 (2011), 314e-321e.

19. Lu, Q, Li Y, Chen T-W, et al: Validity of soft- 
tissue thickness of calf measured using MRI for assessing unilateral lower extremity lymphoedema secondary to cervical and endometrial cancer treatments. Clin. Radiol. 69 (2014), 1287-1294.

20. Beesley, V, M Janda, E Eakin, et al: Lymphoedema after gynecological cancer treatment: Prevalence, correlates, and supportive care needs. Cancer 109 (2007), 2607-2614.

21. Hammond, TM, HN Mayrovitz: Programmable intermittent pneumatic compression as a component of therapy for breast cancer treatment-related truncal and arm lymphedema. Home Hlth. Care Mgmt. Practice (22) 2010, 397-402.

22. Li, K, N Liu, Z Yu, et al: Heating and compression bandage treatment is effective for chronic lymphedema with dermatolymphangioadenitis-a case-controlled study. Lymphat. Res. Biol. (2016), 1-7.

23. Fong, SMM, Ng SSM, WS Luck, et al: Effects of qigong exercise on upper limb lymphedema and blood flow in survivors of breast cancer: A pilot study. Integr. Cancer Ther. 13 (2014), 54-61.

24. Li, LYW, J Chen, N-F Liu: Clinical efficacy of compound prescription of traditional Chinese medicine-lymph fang on treatment of chronic extremity lymphedema. J. Tissue Engineer. Reconstr. Surg. 11 (2015), 185-188.

25. Liu, N-F, W Li, J Chen, et al: Treatment of chronic extremity lymphedema with manual lymph drainage. Chinese J. Plastic Surg. 26 (2010), 337-339.

26. Wittlinger, H, G Wittlinger: Introduction to Dr Vodder's Manual Lymph Drainage. Vol 1: Basic course 4th Edn. Heidelberg:Haug Publishers, 1992.

27. Földi E, M Földi, H Weissleder: Conservative treatment of lymphedema of the limbs. Angiology 36 (1985), 171-178.

28. Mislin, $\mathrm{H}$ : Experimental detection of autochthonous automatism of lymph vessels. Experientia17 (1961), 29-30.

29. Fiaschi, E, G Francesconi, S Fiumicelli, et al: Manual lymphatic drainage for chronic postmastectomy lymphoedema treatment. Panminerva Med. 40 (1998), 48-50.

30. Moseley, AL, M Esplin, NB Piller, J Douglass: Endermologi with and without compression bandaging--a new treatment option for secondary arm lymphedema. Lymphology 40 (2007), 129-137.
31. Lasinski, B: Complete decongestive therapy for treatment of lymphedema. Semin. Oncol. Nurs. 29 (2013), 20-27.

32. Elwell, R: An overview of the use of compression in lower-limb chronic oedema. Br. J. Community Nurs. 21 (2016), 36-42.

33. Williams, A, J Venables: Skin care in patients with uncomplicated lymphoedema. J. Wound Care 5 (1996), 223-226.

34. Douglass, J, P Graves, S Gordon: Self-care for management of secondary lymphedema: A systematic review. PloS Negl. Trop. Dis. 10 (2016), 1-20.

35. Grabenbauer, A, AJ Grabenbauer, $R$ Lengenfelder, et al: Feasibility of a 12-monthexercise intervention during and after radiation and chemotherapy in cancer patients: impact on quality of life, peak oxygen consumption, and body composition. Radiat. Oncol. 11 (2016), 1-7.

36. Williams, AF, A Vadgama, PJ Franks, et al: A randomized controlled crossover study of manual lymphatic drainage therapy in women with breast cancer-related lymphoedema. Eur. J. Cancer Care 11 (2002), 254-261.

37. Weiss, JM, BJ Spray: The effect of complete decongestive therapy on the quality of life of patients with peripheral lymphedema. Lymphology 35 (2002), 46-58.

38. Takeno, Y, H Arita, E Fujimoto: Efficacy of complete decongestive therapy (CDT) on edematous rat limb after lymphadenectomy demonstrated by real time lymphatic fluid tracing. Springer Plus 2 (2013), 225-231.

39. Leduc, O, A Leduc, P Bourgeois, JP Belgrado: The physical treatment of upper limb edema. Cancer 83(1998), 2835-2839.

\author{
Di Sun, PhD \\ Department of Plastic \& Reconstructive \\ Surgery \\ Shanghai Ninth People's Hospital \\ Shanghai Jiao Tong University \\ School of Medicine, \\ 639 Zhi Zao Ju Road \\ Shanghai, 200011, China \\ E-mail: sundi1980hn@sina.com \\ Tel: 15903617756.
}

